\title{
Influence of the Form of Porous Insert in the Flow of a Viscous Liquid in a Plane Pipe
}

\author{
Umurdin Dalabaev \\ University of World Economy and Diplomacy, Tashkent, Uzbekistan \\ Email: udalabaev@mail.ru
}

How to cite this paper: Dalabaev, U. (2022) Influence of the Form of Porous Insert in the Flow of a Viscous Liquid in a Plane Pipe. Journal of Applied Mathematics and Physics, 10, 372-378. https://doi.org/10.4236/jamp.2022.102029

Received: December 11, 2021

Accepted: February 18, 2022

Published: February 21, 2022

Copyright ( 2022 by author(s) and Scientific Research Publishing Inc. This work is licensed under the Creative Commons Attribution International License (CC BY 4.0).

http://creativecommons.org/licenses/by/4.0/ (c) (i) Open Access

\begin{abstract}
Filtration of an incompressible liquid (gas) in a non-deformable porous medium is investigated. The results of numerical simulation of the hydrodynamic features of the flow arising after the passage of the liquid through a layer of an immobile porous medium are presented. An interpenetrating model of multiphase media is used to describe such flows. Kozeny-Karman relations are used as the interaction force. The influence of the geometrical shape of the bulk layer on the nature and magnitude of the inhomogeneity of the flow velocity around the obstacle is shown. The shape of the porous medium significantly affects the flow parameters. Numerical simulation results are compared with experimental data. The shape of the porous medium significantly affects the flow parameters. Numerical simulation results are compared with experimental data. The effects of non-uniformity of the fluid velocity field arising due to the shape of the layer surface are investigated by the methods of a computational experiment. A qualitative comparison is made of velocity inhomogeneities when a fluid flows through a porous obstacle. For the numerical implementation of the filtration equation of the interpenetrating model, a SIMPLE-like algorithm was used.
\end{abstract}

\section{Keywords}

Filtration, Rahmatulin Equation, Fluid-Porous Interface

\section{Introduction}

In natural phenomena and processes, the interaction of a liquid or gas flow with heterogeneous media is observed. The term heterogeneous medium is understood as a combined area in which there is a free zone and a porous layer. One of the types of such interactions is the flow of a flow through a layer of a porous medium. The study of the laws of flow of continuous media through a stationary granular layer is one of the areas of research in the field of mechanics of multi- 
phase media [1]-[10].

In industry, processes of this kind are present in chemical catalytic reactors. When simulating hydrodynamic processes in a layer of a granular medium, the layer creates significant hydrodynamic resistance. In this case, directly behind the layer, a flow inhomogeneity is formed. Such inhomogeneities have been noted in a number of experimental works, for example, in [11] [12] [13].

In [14], the problem of the flow of an ideal incompressible fluid along a flat surface with a stationary granular layer perpendicular to it is considered. The problem is solved by using the self-consistent field method. In the first approximation in terms of the volume fraction of granules in a layer, functions describing the ensemble-averaged liquid velocity profile both inside and outside this layer are obtained in an analytical form. The work [15] is devoted to the influence of the curvature of the granular layer on the structure of the viscous flow. In this case, an " $M$ "-shaped profile of the velocity behind the layer was obtained. Numerical results are obtained based on the large particle method. The work [16] is also devoted to the detection of the profile of inhomogeneities. In this work, the control volume method with a quadratic force of interaction was used in a two-dimensional computational domain. The study of the force of interaction in the form $\boldsymbol{F}=4.5 \mu f(1-f) a^{-2} \boldsymbol{u}$ was carried out in [17] ( $a$ is the radius of the particles of the granular layer). In [18], numerical results were obtained for the problem of flow through a fixed porous layer on the basis of an interpenetrating model with an interaction force in the form of Kozeny-Karman. The non-uniformity of the longitudinal velocity field behind the porous layer has been studied. In this case, the left boundary of the layer was not deformed, and the influence of the right boundary of the layer on the flow structures was investigated.

This work is devoted to the study of the processes of redistribution of the fluid flow in a layer of a stationary granular medium with various types of layer arrangement while observing the interaction force in the Kozeny-Karman form.

\section{Mathematical Model and Numerical Method}

Let us consider an interpenetrating model describing the flows of two-phase media [18] [19], where the velocity of the discrete phase is neglected. Then the flow of the liquid phase is described by a system of equations (two-dimensional case):

$$
\begin{aligned}
& f u \frac{\partial u}{\partial x}+f v \frac{\partial u}{\partial y}=-\frac{f}{\operatorname{Re}} \frac{\partial p}{\partial x}+\frac{4}{3 \operatorname{Re}} \frac{\partial}{\partial x}\left(f \frac{\partial u}{\partial x}\right)+\frac{1}{\operatorname{Re}} \frac{\partial}{\partial y}\left(f \frac{\partial u}{\partial y}\right) \\
& -\frac{2}{3 R e} \frac{\partial}{\partial x}\left(f \frac{\partial v}{\partial y}\right)+\frac{1}{R e} \frac{\partial}{\partial y}\left(f \frac{\partial v}{\partial x}\right)-C u, \\
& f u \frac{\partial v}{\partial x}+f v \frac{\partial v}{\partial y}=-\frac{f}{R e} \frac{\partial p}{\partial y}+\frac{1}{R e} \frac{\partial}{\partial x}\left(f \frac{\partial v}{\partial x}\right)+\frac{4}{3 R e} \frac{\partial}{\partial y}\left(f \frac{\partial u}{\partial y}\right) \\
& +\frac{1}{R e} \frac{\partial}{\partial x}\left(f \frac{\partial v}{\partial y}\right)-\frac{2}{3 R e} \frac{\partial}{\partial y}\left(f \frac{\partial v}{\partial x}\right)-C v \text {. }
\end{aligned}
$$




$$
\frac{\partial(f u)}{\partial x}+\frac{\partial(f v)}{\partial y}=0
$$

Here, $u, v$ is the component of the flow velocity, $p$ is fluid pressure, $f$ is volume concentration, $R e$ is the Reynolds number, $C$ is interaction coefficient. In Equations (1)-(3), the parameters are dimensionless $(R e=U H \rho / \mu), U$ is average volume velocity, $L$ is characteristic scale, $\rho$ is fluid density, $\mu$-viscosity). For the interaction coefficient, the Kozeny-Karman relation was used: $C=\frac{D^{2}}{\operatorname{Re}} \frac{(1-f)^{2}}{f^{2}}$, $D=\sqrt{\alpha} H / d, d$ is the characteristic size of the porous medium.

Equations (1)-(3) allow one to study flows both inside and outside porous media, since at $f=1$ we obtain the Navier-Stokes equations for an incompressible fluid. Moreover, these equations are suitable for the entire area under consideration. For the numerical solution of (1)-(3), we use the control volume method [20] [21] with a non-uniform mesh. The irregular mesh was built so that their thickening was formed around the porous insert. The SIMPLE algorithm [20] is generalized for Equations (1)-(3).

At the boundaries of the computational domain, the adhesion condition is set on a solid wall; constant pressure and Poiselle flow are given at the channel inlet, and the output is a soft boundary condition [17].

\section{Results and Discussion}

1) Gas flow through a rectangular porous barrier. Let a part of the flat channel be filled with a granular medium. Equation (1)-(3) is considered in the area: $0 \leq x \leq L, 0 \leq y \leq 1$. The $x$-axis is directed along the lower wall of the channel, and the $y$ axis is perpendicular to it. At the entrance to the channel, a symmetric velocity profile is given, which makes it possible to consider the problem in the lower half of the channel with the condition of symmetry of the flow at $x=0.5$ $(\partial u / \partial y=0)$. Part of the channel is filled with a granular medium with a porosity of 0.6 .

It can be seen from the graph in Figure 1 that a uniform flow is observed near the exit from the porous layer in the main part, except for the boundary layer where a profile of the boundary layer type is observed. At Reynolds numbers 10 and 100 , the velocity profile near the exit of the porous layer is observed with a maximum profile near the boundary. When passing through the porous layer, the maximum approaches the walls. Streamline graph Figure 2 shows the symmetry of the streamline relative to a rectangular porous layer.

2) Fluid flow through a porous layer with a shift on the channel axis. Let the porous layer in the delicate part of the channel be filled in the area $\Omega=\{(x, y): y+1 \leq x \leq y+1,5 ; 0 \leq y \leq 0.5\}$, while in the upper part it is symmetrical about the axis of the channel.

The calculation results are shown in Figure 3. In this case, the maximum value of the longitudinal velocity is observed near the wall behind the layer (Figure 3). 


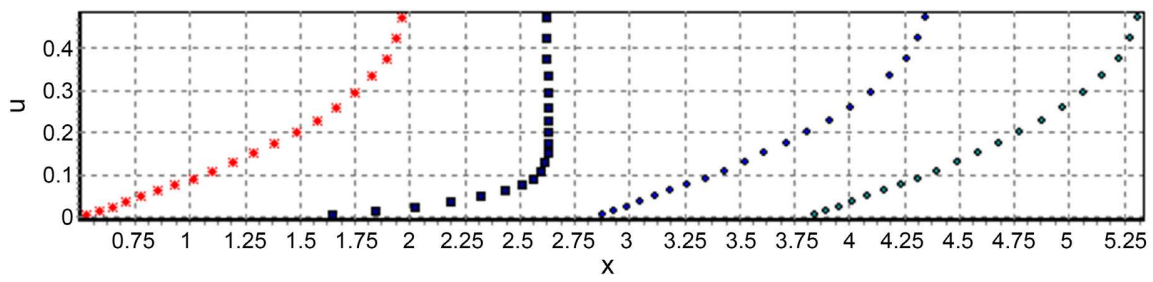

Figure 1. Filtration longitudinal velocity profiles for different channel sections: $x=0.5$; $1.548 ; 2.848$; 3.816 and with parameters: $D=100 ; R e=1$.

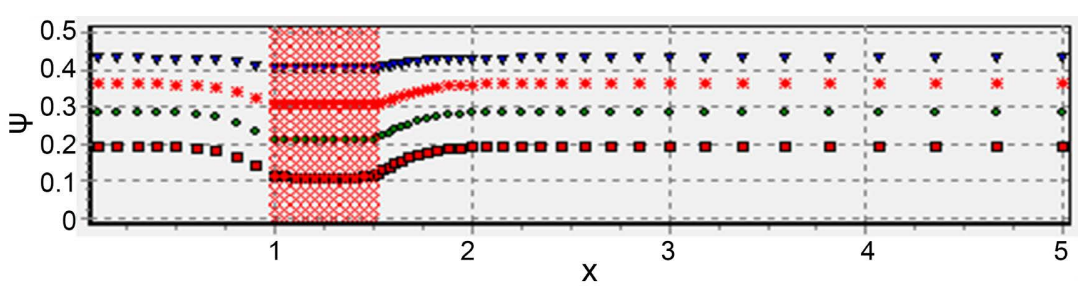

Figure 2. Streamlines at $\psi=0.1 ; 0.2 ; 0.3 ; 0.4$.

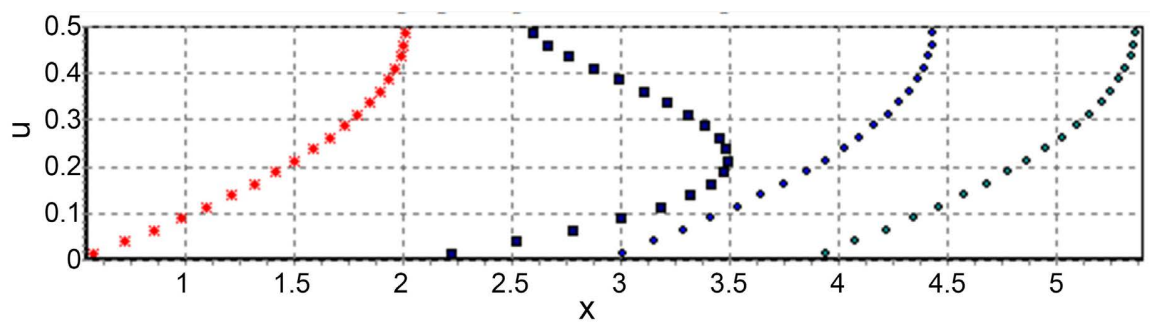

Figure 3. Filtration llongitudinal velocity profiles for different channel sections: $x=0.5$; $2.059 ; 2.905 ; 3.863$; and with parameters: $D=100 ; R e=1$.

The streamline given in Figure 4 clearly shows that, if an increase in velocity is observed behind the layer, and vice versa before the layer. This conclusion coincides with the conclusion of [16], where it is stated that the direction of the fluid flow at each point of the porous region is opposite to the pressure gradient vector. From this, it follows that what type of interaction force is not chosen, an increase in the velocity near the wall is observed.

3) Liquid flow through a porous layer with a shear upstream. Let the porous layer in the delicate part of the channel be filled in the area $\Omega=\{(x, y): 1-y \leq x \leq 1,5-y ; 0 \leq y \leq 0.5\}$, while in the upper part it is symmetrical about the axis of the channel. In this case, the flow structure changes in its own way.

The calculation results are shown in Figure 5. In this case, the maximum value of the longitudinal velocity is observed near the wall in front of the layer (Figure 5). This fact is also confirmed on the basis of the current shown in Figure 6.

4) Liquid flow through a porous layer with a curved surface. Let us consider the case in which a part of the computational domain:

$$
\Omega=\left\{(x, y): \sqrt{2.5-(y-1.5)^{2}} \leq x \leq 0.5+\sqrt{2.5-(y-1.5)^{2}} ; 0 \leq y \leq 0.5\right\} .
$$




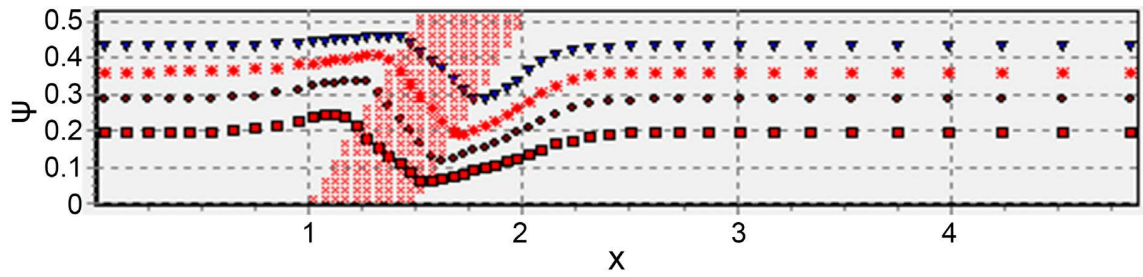

Figure 4. Streamlines at $\psi=0.1 ; 0.2 ; 0.3 ; 0.4(D=100 ; R e=1)$.

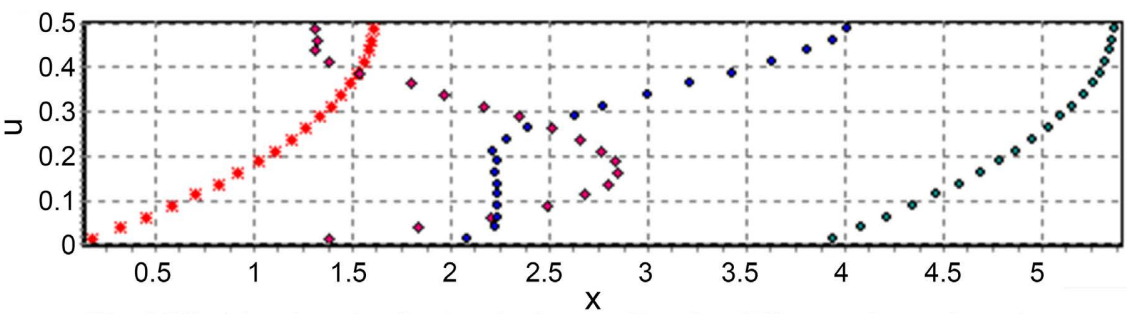

Figure 5. Filtrition longitudinal velocity profiles for different channel sections: $x=0.1$; $1.25 ; 1.75 ; 3.863$; and with parameters: $D=100 ; R e=1$.

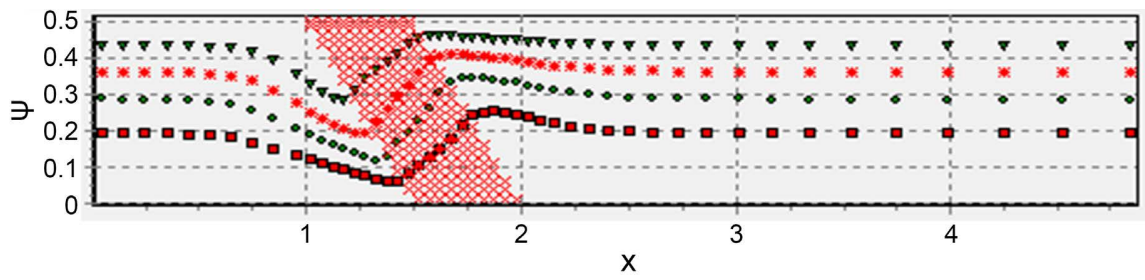

Figure 6. Streamlines at $\psi=0.1 ; 0.2 ; 0.3 ; 0.4$.

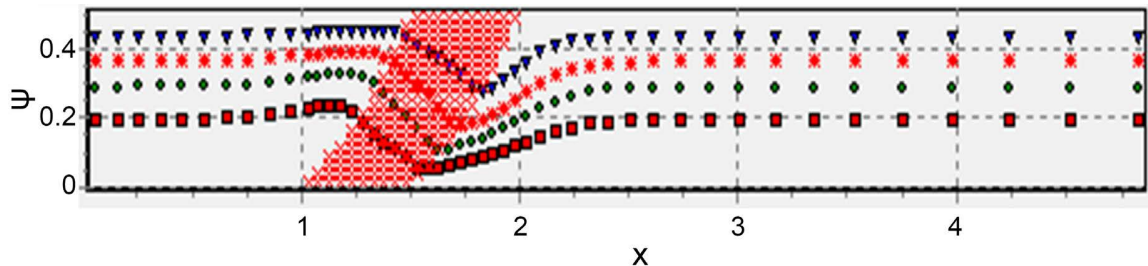

Figure 7. Streamlines at $\psi=0.1 ; 0.2 ; 0.3 ; 0.4$.

is filled with a porous medium and the upper part is symmetrical about the axis of the channel. In this case, the inhomogeneity is observed more noticeably in comparison with the straight boundary (Figure 7).

\section{Conclusions}

Rakhmatulin's model explains the reason for the non-uniformity of the flow velocity profile behind the layer and when observing the interaction force in the Kozeny-Karman form.

The results of the study are in good qualitative agreement with the experimental results.

When the porosity of the layer is constant, the non-uniformity of the longitudinal velocity behind the layer mainly arises due to its geometrical arrangement.

If the shear of the porous layer takes place downstream, then the maximum 
value of the velocity is observed behind the layer near the wall; if the shear of the layer is located upstream, then the maximum value of the velocity is observed in front of the layer.

The main reason for the nonuniformity of the velocity profile behind the layer is the movement of the liquid towards the side with the least resistance, i.e. perpendicular to the layer.

\section{Conflicts of Interest}

The author declares no conflicts of interest regarding the publication of this paper.

\section{References}

[1] Nield, D.A. and Bejan, A. (2017) Convection in Porous Media. Springer International Publishing AG, Berlin. https://doi.org/10.1007/978-3-319-49562-0

[2] Ehrhardt, M. (2000) An Introduction to Fluid-Porous Interface Coupling. Progress in Computational Physics, 2, 3-12. https://doi.org/10.2174/978160805254711201010003

[3] Moralesa, F.A. and Showalterb, R.E. (2017) A Darcy-Brinkman Model of Fractures in Porous Media. Journal of Mathematical Analysis and Applications, 452, 1332-1358. https://doi.org/10.1016/j.jmaa.2017.03.063

[4] Beavers, G.S. and Joseph, D.D. (1967) Boundary Conditions at a Naturally Permeable Wall. Journal of Fluid Mechanics, 30, 197-207. https://doi.org/10.1017/S0022112067001375

[5] Saffman, P.G. (1971) On the Boundary Condition at the Surface of a Porous Medium. Studies in Applied Mathematics, 50, 93-101.

https://doi.org/10.1002/sapm197150293

[6] Ochoa-Tapia, J.A. and Whitaker, S. (1995) Momentum Transfer at the Boundary between a Porous Medium and a Homogeneous Fluid-I. Theoretical Development. International Journal of Heat and Mass Transfer, 38, 2635-2646. https://doi.org/10.1016/0017-9310(94)00346-W

[7] Ochoa-Tapia, J.A. and Whitaker, S. (1998) Momentum Jump Condition at the Boundary between a Porous Medium and a Homogeneous Fluid: Inertial Effect. Journal of Porous Media, 1, 201-217. https://doi.org/10.1615/JPorMedia.v1.i1.30

[8] Yu, P., et al. (2007) A Numerical for Flows in Porous and Homogenous Fluid Domains Coupled at the Interface by Stress Jump. International Journal for Numerical Methods in Fluids, 53, 1755-1775. https://doi.org/10.1002/fld.1383

[9] Iliev, O. and Laptev, V. (2004) On Numerical Simulation of Flow through Oil Filters. Computing and Visualization in Science, 6, 139-146. https://doi.org/10.1007/s00791-003-0118-8

[10] Gol'dshtik, M.A. (1984) Transport Processes in the Granular Layer. Institut Teplofiziki SO AN SSSR, 164 p. (In Russian)

[11] Popov, E.K., Smirnov, E.V., Abaev, G.M., Shtern, P.G., Turuntaev, S.V. and Lychagin, V.F. (1976) Issues of Research of Reactors with a Fixed Bed of Catalyst. Aerodynamics of Chemical Reactors, Novosibirsk, 65-71.

[12] Kirillov, V.A., Kuzmin, V.A., Pyanov, V.I. and Khanaev, V.M. (1979) On the Velocity Profile in a Fixed Granular Layer. DAN SSSR, 245, 159-162.

[13] Strizhov, A.A. and Khalilov, V.Sh. (1994) Structure of Wall Flow through a Channel 
with a Granular Bed. Fluid Dynamics, 29, 745-748.

https://doi.org/10.1007/BF02040780

[14] Guskov, O.B. (2020) The Flow of an Ideal Fluid through a Stationary Granular Layer in the Presence of a Flat Wall. DAN, Physics, Technical Sciences, 491, 37-43.

[15] Mikhailenko, K.I. and Kuleshov, V.S. (2005) Mathematical Modeling of Gas Flow Velocity Irregularity behind a Porous Barrier. Computational Technologies, 20, 46-58.

[16] Mikhailenko, K.I. and Urmantsev, S.F. (2003) Numerical Study of the Flow of a Mixture of Reacting Gases in an Inhomogeneous Catalyst Layer. Proceedings of the Institute of Mechanics of Ufa Branch of RAS, 3, 246-254.

https://doi.org/10.21662/uim2003.1.018

[17] Dalabaev, U. (1997) Structure of Flow through an Immovable Granular Layer. Journal of Engineering Physics and Thermophysics, 70, 379-382. https://doi.org/10.1007/BF02662134

[18] Faizullaev, D.F. (1969) Laminar Motion of Multiphase Media in Conduits. Springer, Berlin. https://doi.org/10.1007/978-1-4899-4830-4_1

[19] Nigmatulin, R.I. (1978) Fundamentals of the Mechanics of Heterogeneous Media. Izdatel'stvo Nauka, Moscow, 336 p. (In Russian)

[20] Patankar, S. (1980) Numerical Heat Transfer and Fluid Flow. CRC Press, Boca Raton.

[21] Blazek, J. (2001) Computational Fluid Dynamics: Principles and Applications. Elsevier, Amsterdam, 470. 